\title{
A Unidirectional Insulated AC-DC Converter Based on the Hexverter and Multipulse-Rectifier
}

\author{
Gustavo Lambert, Student Member, IEEE, Alessandro Costabeber, Member, IEEE, \\ Pat Wheeler, Senior Member, IEEE, and Yales R. De Novaes, Member, IEEE
}

\begin{abstract}
This paper proposes an insulated two-stage ac-dc converter to be applied in unidirectional power flow systems. The two-stage structure is the combination of a Hexverter and a series-type 12 pulse rectifier. The key features of the proposed structure are inherited from the modular multilevel converter concept such as modularity, scalability, and expandability. Besides the topology, this article presents a complete control strategy based on the output (dc) voltage regulation and Hexverter's stored energy. In particular, an unusual method to regulate the Hexverter's branch energy unbalances is proposed. All currents in the Hexverter are controlled which increases the robustness for operation in harsh environments such as subsea systems. Mathematical analysis of the control loops is carried out to develop the necessary control-oriented models for each control loop. The converter and the control strategy are verified through experiments in a 24 cells Hexverter and a current-fed series-type 12-pulse rectifier.
\end{abstract}

Index Terms-Hexverter, Multipulse Rectifier, ac-dc, Medium Frequency, Current Source.

\section{INTRODUCTION}

$\mathbf{S}$ EVERAL offshore facilities are usually dependent on gas turbines, which operate continuously in a harsh environment to supply power and gas compression for the subsea processing systems. In the harsh environment where these machines are installed issues with the turbine, although not so often, can delay the production and compromise the operation. Then, alternative methods for supplying the system or even restructuring it in a subsea power grid can potentially reduce the cost and complexity of bringing offshore hydrocarbons in a more processed form to the surface. Together or instead of the gas turbines, alternative power supplies as offshore wind farms [1] or energy from onshore, as the Valhall project [2], seem very good alternatives to increase significantly the reliability and availability of the system. In addition, they may reduce the environmental impact of the offshore industry.

Modifications in the power supply of such a subsea power system, however, requires attention not only in the power supply itself but all the elements involved in the power transmission, distribution, and the electronic equipment, as reported in [3]. Due to the harsh environment of a subsea

This work was developed with the support of the Programa Nacional de Cooperação Acadêmica da Coordenação de Aperfeiçamento de Pessoal de Nivel Superior - CAPES/Brazil. The authors also would like to thank FAPESC and UDESC for their financial support.

G. Lambert and Y. R. De Novaes are with the Electrical Power Processing Group, Santa Catarina State University, Joinville, SC, Brazil, 89219-710, Brazil. e-mail: gustavolambert@outlook.com; yales.novaes@udesc.br.

A. Costabeber and P. Wheeler are with the PEMC group, University of Nottingham, NG72RD, Nottingham, UK. power system (very high pressures and high temperatures), there is a concern to all the various power components such as pressure tolerant power electronics, high-voltage circuitbreakers, power umbilical, wet-mate connector, and other. Moreover, such systems are expected to operate for a long period without failure or even maintenance since the access to the equipment is difficult due to the distance and pressure involved. Consequently, one of the main key features of subsea equipment is reliability [4].

Among the possible arrangements a subsea power system could have, this paper is focused on the unidirectional powerflow arrangement as shown in Fig. 1. Also, for the distances involved, it is assumed that the ac line is economically and technically viable [5]. Then, the choice for ac lines benefits from the developed protection mechanisms and equipment. For the power loads, it is considered that the majority are driven by a Variable Speed Drive (VSD) fed by the dc distribution lines. The later removes the need for a rectification stage commonly found in VSDs and, ideally, avoid the energy quality problems related to the ac lines, such as resonances and harmonics circulation [6]. For such a scenario, this paper address a power electronic converter topology that enables the connection between the ac power line and the dc loads (ac-dc block in Fig. 1). Due to the power levels (MW) and distance, this converter is expected to operate with ac input in the tens of $\mathrm{kV}$ range while the dc output may range in the units of $\mathrm{kV}$. For this task few are the power electronics means to process the energy without the use of low-frequency transformers. Recently, the more successful results have been achieved by the modular multilevel converters, which developments in its family are reviewed in [7].

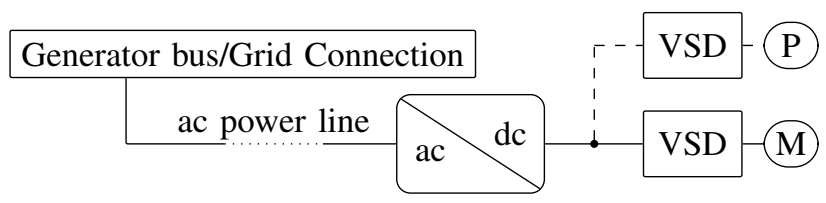

Fig. 1. Simplified power system block diagram for ac transmission and dc distribution. The Variable Speed Drives (VSD) main loads are high-power pumps, compressors and drilling machines.

Nevertheless, the isolation feature is important for the operation, thus a transformer must be incorporated into the structure. One possibility is to create a mix of modular multilevel converters and low-frequency transformers [8], [9]. Another alternative is to operate the transformer with a frequency higher than the ac power line. This could be achieved by 
modifying the ac-dc structure into an ac(low frequency)ac(medium frequency)-dc one. Such a link would be a design variable and could be used to reduce the size of the transformer and the energy variation in the modular multilevel converter. Among the modular multilevel converters able to create this ac link, the main alternatives are the back-to-back MMC (BTBMMC), the Modular Multilevel Matrix Converter (MMMC) and the Hexverter. Although comparisons have shown that the BTB-MMC and the MMMC as usually superior to the Hexverter [10], [11], in this application it might not be true. Since there is no frequency lower than the grid where highpower is required, the main advantages for the MMMC are not seen. In the case of the BTB-MMC, there is a chance that for increased blocking capabilities the full-bridge submodules are required instead of half-bridge, which would increase greatly the number of elements. Then, although just slightly better, the Hexverter is chosen among the other due to its blocking features and a smaller number of elements, which includes not only the branches but also auxiliary circuits and sensors. Not only the second ac link, but also the use of a solid-state active converter enables stable operation, within limits, even under severe voltage or frequency variations. Furthermore, other functionalities could be incorporated in this active converter, e.g. the ac line reactive power compensation.

Considering unidirectional operation, the multipulse rectifier class is selected for the ac-dc conversion [12]. Using this rectifier not only isolation is achieved but also a reduced amount of not active energy circulation and the feasibility of lower voltage rectifier bridges connected in series to reach MVDC.

As a result, this paper presents the two-stage arrangement composed of an ac-ac converter and an isolated multipulse rectifier. More specifically, this is realized by the Hexverter and the series-type 12-pulse rectifier arrangement. In addition to the structure, the analysis and development of the control strategies are also shown. The converter's operation and control strategy are presented along with necessary mathematical analysis to obtain control-oriented models. The proposed Hexverter's branch energy regulation loops are based on the works of [13], [14]. But in our approach, we use information from the submodule's voltages, decomposed by the $\alpha \beta 0$ transformation, to generate the circulating currents references for the branch energy control. Furthermore, the Herverter's input currents are defined by the stored energy and the Hexverter's output currents are set by the 12-pulse rectifier in an inner loop for the output voltage control. Experimental results are shown to verify the converter operation and to test the proposed control strategy.

\section{HeXverter Elements AND Definitions}

The Hexverter, first proposed in [15] and further discussed in [16], is a modular multilevel converter able to perform three-phase to three-phase ac-ac conversion. This converter is composed of six branches connected in series where each branch has a cascade connection of $\mathrm{N}$ submodules (SMs). For ac-ac operation, each SM is required to be bi-directional both in voltage and current. Because of it, the most common choice is the full-bridge converter. As other modular multilevel converters, inductors $\left(L_{b}\right)$ are inserted in series with each branch to limit the current derivative during the submodule switching process. According to [17], the Hexverter is classified as Double-Delta Bridge-Cells (DDBC). Further details on the topology can be found in [11].

The ideal Hexverter has two different three-phase threewire systems (system 1 and system 2 as shown in Fig. 2. For the converter analysis the voltage and current references are defined according to Fig. 2. Then, assuming no current circulation in the branches, by the Kirchhoff voltage law the each branch voltages $\left(v_{b, m}, m=\{1,2 \ldots, 6\}\right)$ as function of system $1\left(v_{1, k}, k=\{1,2,3\}\right)$ and system 2 voltages $\left(v_{2, k}\right.$, $k=\{1,2,3\})$ are

$$
\left[\begin{array}{l}
v_{b, 1} \\
v_{b, 2} \\
v_{b, 3} \\
v_{b, 4} \\
v_{b, 5} \\
v_{b, 6}
\end{array}\right]=\left[\begin{array}{ccccccc}
1 & 0 & 0 & -1 & 0 & 0 & 1 \\
0 & -1 & 0 & 1 & 0 & 0 & -1 \\
0 & 1 & 0 & 0 & -1 & 0 & 1 \\
0 & 0 & -1 & 0 & 1 & 0 & -1 \\
0 & 0 & 1 & 0 & 0 & -1 & 1 \\
-1 & 0 & 0 & 0 & 0 & 1 & -1
\end{array}\right] \cdot\left[\begin{array}{c}
v_{1,1} \\
v_{1,2} \\
v_{1,3} \\
v_{2,1} \\
v_{2,2} \\
v_{2,3} \\
v_{s t}
\end{array}\right]
$$

where $v_{s t}$ is the voltage difference between system 1 and system 2 center node, which is named star-voltage. Due to the

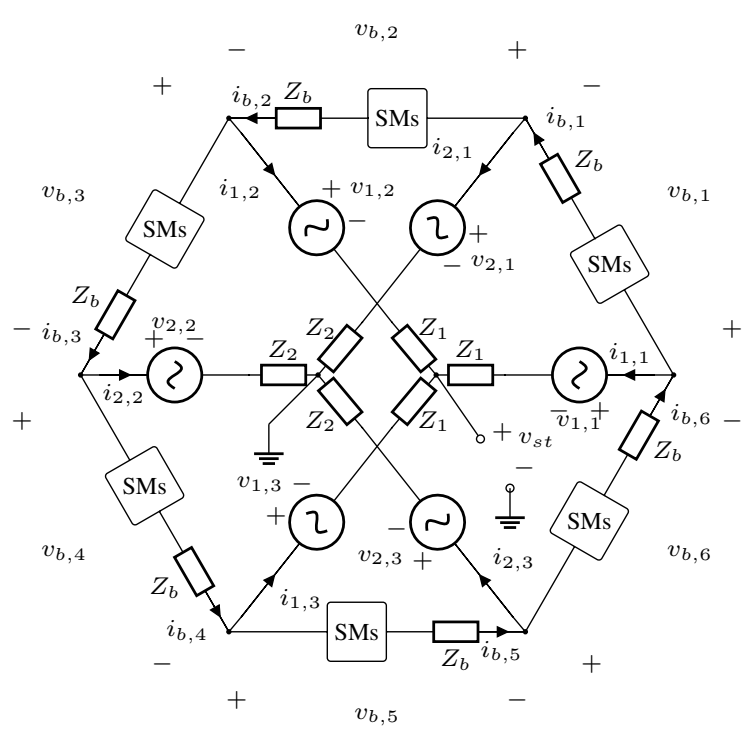

Fig. 2. Heverter voltage and current references for the sources $\left(v_{x, y}\right.$ and $\left.i_{x, y}\right)$ and branches $\left(v_{b, m}\right.$ and $\left.i_{b, m}\right)$.

coupling existent in a three-phase three-wire system, these set of equations can be simplified by the use of $\alpha \beta$ transformation into a decoupled two variables system.

$$
\left[\begin{array}{l}
x_{\alpha} \\
x_{\beta}
\end{array}\right]=\left[T_{\alpha \beta}\right] \cdot\left[\begin{array}{l}
x_{1} \\
x_{2} \\
x_{3}
\end{array}\right]=\left[\begin{array}{ccc}
2 / 3 & -1 / 3 & -1 / 3 \\
0 & 1 / \sqrt{3} & -1 / \sqrt{3}
\end{array}\right] \cdot\left[\begin{array}{l}
x_{1} \\
x_{2} \\
x_{3}
\end{array}\right]
$$

Using the $T_{\alpha \beta}$ and (1), the branch voltages can be rewritten as (3). In this representation of the converter voltages, the addition of a common voltage $\left(v_{\text {sum }}\right)$ to all branch voltages is suggested in [15]. This voltage is defined as $v_{\text {sum }}=\sum_{m=1}^{6} v_{b, m}$ 
and used to control the circulating current $i_{\text {circ }}$, which is defined as $i_{c i r c}=\sum_{m=1}^{6} i_{b, m} / 6$.

$$
\left[\begin{array}{l}
v_{b 1} \\
v_{b 2} \\
v_{b 3} \\
v_{b 4} \\
v_{b 5} \\
v_{b 6}
\end{array}\right]=\left[\begin{array}{cccccc}
1 & 0 & -1 & 0 & 1 & 1 / 6 \\
1 / 2 & -\sqrt{3} / 2 & 1 & 0 & -1 & 1 / 6 \\
-1 / 2 & \sqrt{3} / 2 & 1 / 2 & -\sqrt{3} / 2 & 1 & 1 / 6 \\
1 / 2 & \sqrt{3} / 2 & -1 / 2 & \sqrt{3} / 2 & -1 & 1 / 6 \\
-1 / 2 & -\sqrt{3} / 2 & 1 / 2 & \sqrt{3} / 2 & 1 & 1 / 6 \\
-1 & 0 & -1 / 2 & -\sqrt{3} / 2 & -1 & 1 / 6
\end{array}\right] \cdot\left[\begin{array}{c}
v_{1, \alpha} \\
v_{1, \beta} \\
v_{2, \alpha} \\
v_{2, \beta} \\
v_{s t} \\
v_{\text {sum }}
\end{array}\right]
$$

Hexverter's operation requires each branch to synthesize voltages with the frequency of system 1 and system 2 . The operation of the Hexverter focus on the balancing of system 1 and system 2 voltages by the branch voltages. Then, the voltage of each adjacent branch pair must at least balance one system's line voltage. For instance, the adjacent pair voltage $v_{b, 1}+v_{b, 2}$ exchange power with the line voltage $v_{1,2}-v_{1,1}$ while the adjacent pair $v_{b, 2}+v_{b, 3}$ exchange power with the line voltage $v_{2,2}-v_{2,1}$. The use of (1) or (3), naturally results in a correct match of branch voltages and the voltages from system 1 or system 2. For example, the resultant voltage of $v_{b, 1}+v_{b, 2}$ voltage has a frequency $f_{1}$ and the resultant voltage of $v_{b, 4}+v_{b, 5}$ has a frequency $f_{2}$.

\section{AC-DC CONVERTER Operation AND CONTROL}

The proposed converter is shown in Fig. 3. The first stage (Hexverter) converts the input (system 1) ac into output (system 2) ac which has a higher frequency. The second stage is the isolated series-type 12-pulse rectifier with capacitive filter. Regardless of the control strategy, the ac-ac converter is meant to be operated as a balanced three-phase sinusoidal current source to feed the 12-pulse rectifier, accordingly to the approach presented in [12]. This operation mode presents advantages as the ac side inherited short-circuit protection, transformer's voltage step limited by the output rectifier voltage and current limiting during overloads.

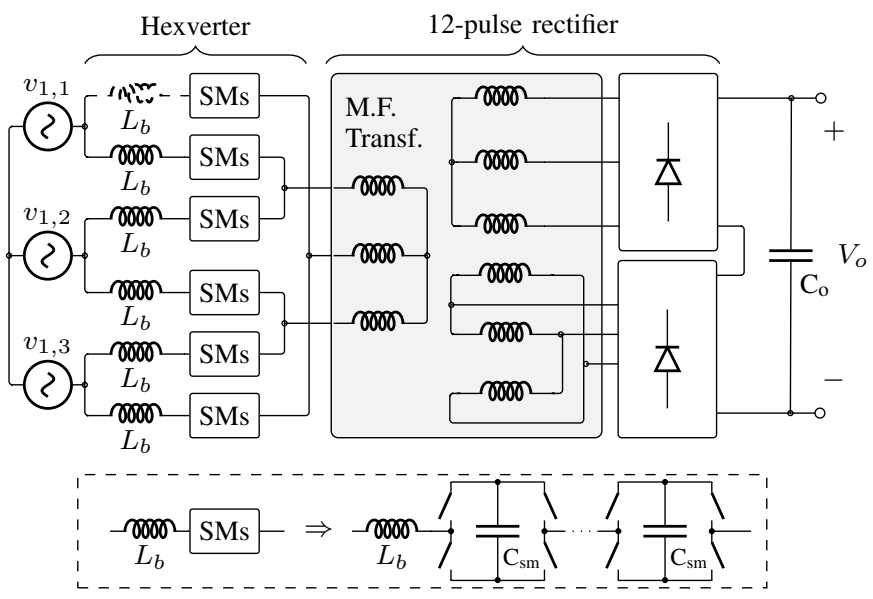

Fig. 3. Proposed ac-dc structure composed by a Hexverter and a 12-pulse series type rectifier. Each Hexverter's branch is composed of an inductor and a group of full-bridge converters connected in cascade (SMs).

In compass with the operation, the control strategy is required to control the output voltage and the Hexverter's stored energy. This is realized by a central unit, which limits the modularity and expandability but is less complex than a distributed strategy [18]. These requirements are addressed by the control strategy through three external control loops: the output voltage control, the total voltage control and the branch energy regulation loop. In comparison with [14], [16], the work in this paper does not set the input, output or branch power directly. Here the Hexverter's powers (input, output or branch) are set indirectly by the output voltage, by the SMs total voltage and by the branches total voltages differences. This approach was chosen to give direct control over the variables of interest.

\section{A. Output voltage control}

The output voltage control is composed by a cascaded of two loops as shown in the block diagram of Fig. 4. The inner loop controls the 12-pulse rectifier input current, which are the currents of system 2 . These sinusoidal currents references are a orthogonal set $\left(i_{2, \alpha}^{*}\right.$ and $\left.i_{2, \beta}^{*}\right)$ that is modulated in amplitude by the outer loop control action $\left(u_{v o}\right)$ in the block $u_{v o} / \alpha \beta$.

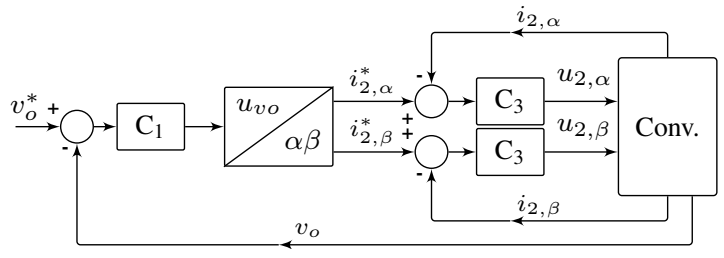

Fig. 4. Block diagram of the output voltage $\left(v_{o}\right)$ control and system 2 current $\left(i_{2, \alpha \beta}\right)$ control.

\section{B. Total voltage control}

This loop indirectly controls the Hexverter's stored energy by the outer loop. For the sake of simplicity, the control variable is set to be the sum of all SM capacitor voltages. In the inner loop of this cascaded strategy, the input currents are in-phase with system 1 sources and their amplitudes are modulated by the total voltage loop control action $\left(u_{v t}\right)$ in the block $u_{v t} / \alpha \beta$.

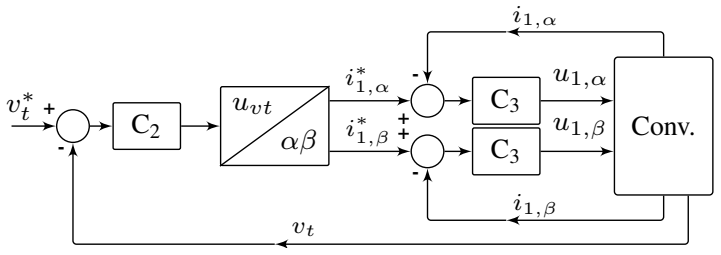

Fig. 5. Block diagram of the total voltage $\left(v_{t}\right)$ control and input current $\left(i_{1, \alpha \beta}\right)$ control.

\section{Proposed Branch Energy Regulation}

As other modular multilevel converters, the Hexverter requires control methods to evenly distribute the stored energy among its SMs. The energy distribution unbalances can be split in two categories. The first occurs among the SMs inside each converter's branch. This is basically due to the 
adopted modulation and differences in losses of each SM. Such unbalance is usually compensated by sorting algorithms [19], [20]. The second is a result of the power absorbed or provided by each branch. This result is exploited in [14], where the authors explain the branch power and suggest how to regulate it by the means of different components in the star voltage $\left(v_{s t}\right)$ and in the circulating current $\left(i_{c i r c}\right)$ which gives several degrees of freedom usable or not accordingly to the Hexverter's operation frequencies in system 1 and 2 .

Following the main principle beyond the branch energy regulation that is to control the branch power in order to charge or discharge it. It is proposed the use of $i_{\text {circ }}$ to regulate the energy distribution between the branches while the $v_{s t}$ remains constant. The $i_{\text {circ }}$ elements are chosen to compensate specific branch energy unbalances. These are monitored by the means of each branch equivalent total voltage $\left(v_{q, m}\right)$, which is the sum of all capacitor's voltages in a given branch $\left(v_{q, m}, m \in\{1,2, \ldots, 6\}\right)$. Although a fixed value of $v_{s t}$ is proposed, it is known from the literature that there is an optimal ratio $v_{s t} / i_{c i r c}$ value for different power situations [21].

The proposed cascaded control loops for the branch energy relation and the circulating current are shown in Fig. 6 and further detailed in Fig. 7. Notice that instead of calculating the required branch power as previously presented by other authors, this strategy uses the information contained in $V_{q, m}$ to generate the circulating current reference $\left(i_{c i r c}^{*}\right)$. Ideally, $i_{\text {circ }}^{*}$ has in its composition a dc element from $u_{v, 1}$, two ac elements with frequency $\omega_{1}\left(u_{v, 2}\right.$ and $u_{v, 3}$ and two ac elements with frequency $\omega_{2}\left(, u_{v, 4}\right.$ and $\left.u_{v, 5}\right)$.

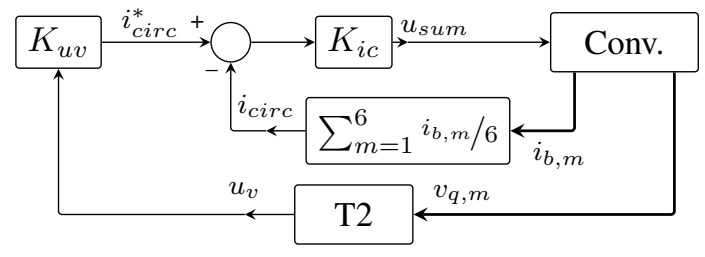

Fig. 6. Branch energy regulation loop and circulating current loop.

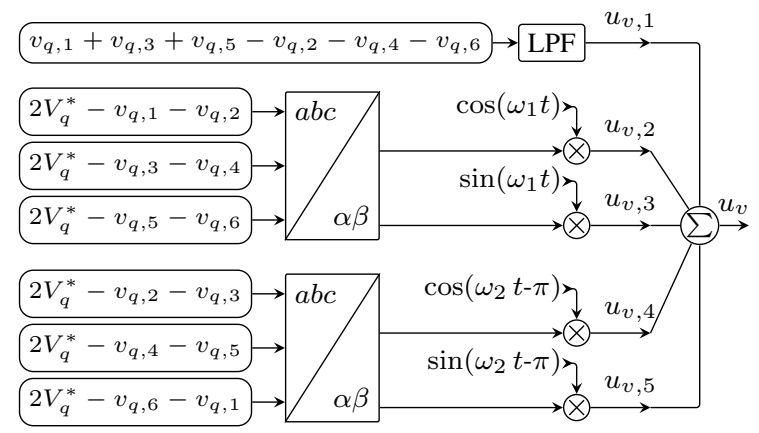

Fig. 7. Block T2 expanded to show the branch equivalent total voltages $\left(v_{q, m}\right)$ and $u_{v, 1} \ldots u_{v, 5}$ relations.

In total, the circulating current reference is meant to be composed of five control actions $\left(u_{v, m} \in\{1,2, \ldots 6\}\right)$. The first portion $\left(u_{v, 1}\right)$ is relative to the adjacent power, which is a consequence of different reactive powers in system 1 and system 2, discussed in detail by [16]. The remaining portions result of unbalances measured by the branches connected to the line voltages of system $1\left(v_{q, 1}+v_{q, 2}, \ldots+v_{q, 6}\right)$ and branches connected to the line voltages of system 2 $\left(v_{q, 2}+v_{q, 3}, \ldots v_{q, 6}+v_{q, 1}\right)$. This information is then modulated by cosines or sines of the respective system frequency, in order to produce an instantaneous branch power that compensates the unbalance.

For a given difference from $v_{q, m}$ reference $\left(N \cdot V_{s m}=V_{q}^{*}\right)$ and the measured voltage, the resultant error can be obtained by $e_{v, m}=V_{q}^{*}-v_{q, m}$. This implies in the following control actions derived as functions of $e_{v, m}$.

$$
\begin{aligned}
& u_{v, 1}=-e_{v, 1}+e_{v, 2}-e_{v, 3}+e_{v, 4}-e_{v, 5}+e_{v, 6} \\
& u_{v, 2}=\cos \left(\theta_{1}\right) \cdot\left[\frac{2\left(e_{v, 1}+e_{v, 2}\right)-e_{v, 3}-e_{v, 4}-e_{v, 5}-e_{v, 6}}{3}\right] \\
& u_{v, 3}=\sin \left(\theta_{1}\right) \cdot\left(\frac{e_{v, 3}+e_{v, 4}-e_{v, 5}-e_{v, 6}}{\sqrt{3}}\right) \\
& u_{v, 4}=-\cos \left(\theta_{2}\right) \cdot\left[\frac{e_{v, 4}+e_{v, 5}+e_{v, 6}+e_{v, 1}-2\left(e_{v, 2}+e_{v, 3}\right)}{3}\right](7) \\
& u_{v, 5}=-\sin \left(\theta_{2}\right) \cdot\left(\frac{-e_{v, 4}-e_{v, 5}+e_{v, 6}+e_{v, 1}}{\sqrt{3}}\right)
\end{aligned}
$$

which results in the circulating current reference

$$
i_{\text {circ }}^{*}=K_{u v} \cdot\left(u_{v, 1}+u_{v, 2}+u_{v, 3}+u_{v, 4}+u_{v, 5}\right) .
$$

An important understanding about the elements $u_{v, 2}, \ldots, u_{v, 5}$ is that the resultant circulating current implies a set of branch power which addition will be zero. This characteristic enhances the stability of the method because the energy extracted from a pair of branches is send to the remaining ones. To prove this statement consider the branches 1 and 2, they are connected in parallel with the line voltage $v_{1,1}-v_{1,2}$. The instantaneous power in this branch combination is

$$
p_{12}=v_{b, 1} \cdot i_{b, 1}+v_{b, 2} \cdot i_{b, 2}
$$

It is known that the branch current has several components, but the interest here is in the power generated by the energy regulating loop. Then, assuming the circulating current control to operate adequately, the remaining power is

$$
p_{12, i c}=\left(v_{b, 1}+v_{b, 2}\right) \cdot i_{c i r c}
$$

which can be rewritten using the $v_{b, m}$ in $\alpha \beta$ coordinates. Also, for the sake of simplicity, the circulating current is supposed to be composed only by $i_{d c}+i_{f_{1}}+i_{f_{2}}$. Due to the superposition principle what is obtained for the system 1 currents $\left(i_{1}\right)$ is also valid for system 2 currents $\left(i_{2}\right)$. Then, for the system 1 frequency, the power produced by the circulating current in the branch pair $1-2$ is

$$
p_{12, i c 1}=\left(v_{b, 1}+v_{b, 2}\right) \cdot K_{u v} \cdot\left(u_{v, 2}+u_{v, 3}\right)
$$

Substituting the branch voltages values but $v_{s t}$ and $v_{\text {sum }}$.

$$
p_{12, i c 1}=\left(\frac{3}{2} v_{1, \alpha}+\frac{\sqrt{3}}{2} v_{1, \beta}\right) \cdot K_{u v} \cdot\left(u_{v, 2}+u_{v, 3}\right)
$$


All the other adjacent branches power can be obtained in the same fashion.

$$
\begin{aligned}
& p_{34, c 1}=\sqrt{3} v_{1, \beta} \cdot K_{u v} \cdot u_{v, 3} \\
& p_{56, c 1}=\left(-3 / 2 \cdot v_{1, \alpha}-\sqrt{3} / 2 \cdot v_{1, \beta}\right) \cdot K_{u v} \cdot\left(u_{v, 2}+u_{v, 3}\right) \\
& p_{23, c 2}=\left(3 / 2 \cdot v_{2, \alpha}-\sqrt{3} / 2 \cdot v_{2, \beta}\right) \cdot K_{u v} \cdot\left(u_{v, 4}+u_{v, 5}\right) \\
& p_{45, c 2}=\sqrt{3} \cdot v_{2, \beta} \cdot K_{u v} \cdot u_{v, 5} \\
& p_{61, c 2}=\left(-3 / 2 \cdot v_{2, \alpha}-\sqrt{3} / 2 \cdot v_{2, \beta}\right) \cdot K_{u v} \cdot\left(u_{v, 4}+u_{v, 5}\right)
\end{aligned}
$$

where $p_{12, c 1}+p_{34, c 1}+p_{56, c 1}=0$ as well as $p_{23, c 2}+p_{45, c 2}+$ $p_{61, c 2}=0$.

The same reasoning applies to $u_{v, 1}$ and the power generated in all branches due to it. For instance, the expected average power in the branch $1\left(\bar{p}_{1, u v 1}\right)$ is

$$
\bar{p}_{1, u v 1}=v_{s t} \cdot K_{u v} \cdot u_{v, 1}
$$

where all six branches will have the same power magnitude. However, branches 1, 3 and 6 differ from branches 2, 4 and 6 in power flow due to $v_{s t}$ polarity.

\section{Control-oriented Models}

For the development of the control-oriented models it is considered that the Hexverter's system 1 and 2 are balanced three-phase voltage sources. Although some characteristics of the 12-pulse rectifier are considered for the output voltage control, the current control of the Hexverter only accounts for the needed power. In terms of energy, the only element storing energy considered is the SM's capacitor. And, each Hexverter branch is assumed to have an equivalent submodule per branch, which voltage and capacitance values are the series equivalent of its SMs.

\section{A. Input, output and circulating current models}

For the proposed control strategy the Hexverter, current models are already available from previous publications as [15], [16], where it is shown the coupling between the Hexverter currents. Since external inductances are inserted to make $L_{b} \ll L_{1}, L_{2}$ and $R_{b} \ll R_{1}, R_{2}$ true. The decoupled currents model presented in [15] is presented in the form of Laplace transfer functions as

$$
\begin{aligned}
\frac{I_{1, \alpha}(s)}{V_{1, \alpha}(s)} & =\frac{I_{1, \beta}(s)}{V_{1, \beta}(s)}=\frac{1}{L_{1} s+R_{1}} \\
\frac{I_{2, \alpha}(s)}{V_{2, \alpha}(s)} & =\frac{I_{2, \beta}(s)}{V_{2, \beta}(s)}=\frac{1}{L_{2} s+R_{2}} \\
\frac{I_{\text {circ }}(s)}{V_{\text {sum }}(s)} & =\frac{1}{6 L_{b} s+R_{b}}
\end{aligned}
$$

where the four $\alpha \beta$ converter voltages $\left(v_{1, \alpha}, \ldots v_{2, \beta}\right)$ are related to the control actions $\left(u_{1, \alpha}, \ldots u_{2, \beta}\right)$ by $v_{x, y}=u_{x, y} \cdot N$. $V_{s m} \cdot k_{p w m}$, where $k_{p w m}$ is the modulator gain, $x \in\{1,2\}$ and $y \in\{\alpha, \beta\}$.

\section{B. Total voltage model}

This model is developed considering the difference between the input power $\left(P_{1}\right)$ and the output power $\left(P_{2}\right)$. Such power difference $\left(\Delta P=P_{1}-P_{2}\right)$ implies in charging or discharging all the Hexverter's submodules. For a balanced three-phase system, the power variation and the converter's stored energy variation are

$$
\Delta P=3 \cdot v_{1} \cdot i_{1}-P_{2}=\frac{\mathrm{d}\left(\Delta W_{T}\right)}{\mathrm{d} t}
$$

where $i_{1}$ and $v_{1}$ are the source's current and phase voltage, respectively. Then, the total energy variation $\left(\Delta W_{T}\right)$ is modeled as the series equivalent of all capacitors in the Hexverter $\left(C_{s m} /(6 \cdot N)\right)$ by

$$
\Delta W_{T}=\frac{C_{s m}}{12 \cdot N} \cdot\left(v_{t f}^{2}-v_{t i}^{2}\right)
$$

where $v_{t f}$ is the final voltage and $v_{t i}$ is the initial voltage. Substituting $v_{t f}=v_{t i}+\Delta v_{t}$ into (24), the $\Delta W_{T}$ becomes

$$
\Delta W_{T}=\frac{C_{s m}}{12 \cdot N} \cdot\left(2 \cdot v_{t i} \cdot \Delta v_{t}+\left(\Delta v_{t}\right)^{2}\right)
$$

If the value $\left(\Delta v_{t}\right)^{2}$ it is assumed small enough to be neglected when compared to $2 \cdot v_{t i} \cdot \Delta v_{t}$, the power variation is

$$
\Delta P=\frac{\mathrm{d}\left(\Delta W_{T}\right)}{\mathrm{d} t}=\frac{C_{s m} \cdot v_{t i}}{6 \cdot N} \cdot \frac{\mathrm{d}\left(\Delta v_{t}\right)}{\mathrm{d} t}
$$

Since the control variable related to the $P_{1}$ is the input current amplitude, (26) is rewritten as

$$
3 \cdot v_{1} \cdot i_{1, p k} / \sqrt{2}=\frac{C_{s m} \cdot v_{t i}}{6 \cdot N} \cdot \frac{\mathrm{d}\left(\Delta v_{t}\right)}{\mathrm{d} t}+P_{2}
$$

where the input current amplitude value assumes a pure sinusoidal wave.

Finally, the transfer function relating the total voltage variation $\left(V_{t}(s)\right)$ and the input current $\left(I_{1, p k}(s)\right)$ is

$$
\frac{V_{t}(s)}{I_{1, p k}(s)}=\frac{18 \cdot v_{1} \cdot N}{\sqrt{2} \cdot v_{t i} \cdot C_{s m} \cdot s}=\frac{18 \cdot v_{1} \cdot N}{\sqrt{2} \cdot 6 \cdot N \cdot v_{s m} \cdot C_{s m} \cdot s}
$$

where $P_{2}$ is assumed to be a perturbation in the model.

\section{Output voltage model}

The transfer function for a 12-pulse rectifier with an input balanced sinusoidal current source has already been presented in [22]. The small-signal model is

$$
\frac{V_{o}(s)}{I_{2, p k}(s)}=\frac{3}{\pi} \cdot \frac{n_{p, y}}{n_{p, y}} \cdot \frac{\left[1+\cos \left(30^{\circ}\right)\right] \cdot \sin \left(15^{\circ}\right) \cdot Z_{o}}{\left(Z_{o} \cdot C_{o} \cdot s+1\right)}
$$

where $I_{2, p k}(s)$ represents the amplitude of a balanced threephase sinusoidal current source reference. The transformer primary and secondary wye windings turns ratio is $n_{p, y} / n_{s, y}$ and the output load impedance is $Z_{o}$. 


\section{Branch energy exchange models}

For the outer loop of the control strategy presented in Fig. 7, there are two transfer functions to be obtained in order to design the gain $K_{u v}$. One is the relation between the voltages difference $\left(v_{q, d i f}=v_{q, 1}+v_{q 3}+v_{q 5}-v_{q 2}-v_{q 4}-v_{q 6}\right)$ and the circulating current reference $\left(i_{v 1}=K_{u v} \cdot u_{v, 1}\right)$. The other is the pairs of voltage differences $\left(2 V_{q}^{*}-v_{q, 1}-v_{q, 2}, \ldots\right)$ and their circulating current references $\left(i_{v 2}=K_{u v} \cdot u_{v, 2}, \ldots\right)$, which even seeming unrelated have all the same dynamic.

Once the power produced due to the circulating current is known by (19), all six branch power equations related to $i_{v 1}$ are added as

$$
6 v_{s t} \cdot K_{u v} \cdot u_{v, 1}=\bar{p}_{1}-\bar{p}_{2}+\bar{p}_{3}-\bar{p}_{4}+\bar{p}_{5}-\bar{p}_{6}
$$

considering an operating point and a small-signal variation $\left(v_{q, 1}=V_{q, 1}+\tilde{i}_{q, 1}\right.$ and $\left.i_{v 1}=I_{v 1}+\tilde{i}_{v 1}\right)$. The use of the capacitor's energy $\left(E_{q 1}=C_{s m} v_{q, 1}^{2} / 2 N\right)$ derivative gives

$$
6 v_{s t} \cdot\left(\tilde{i}_{v 1}+I_{v 1}\right)=\frac{C_{s m}}{2 \cdot N} \cdot \frac{\mathrm{d}\left(2 \cdot V_{q, d i f} \cdot \tilde{v}_{q, d i f}+\tilde{v}_{q, d i f}^{2}\right)}{\mathrm{d} t}
$$

If the second order $\left(\tilde{v}_{q, d i f}^{2}\right)$ and dc $\left(I_{v 1}\right)$ elements are neglected, the use of Laplace's transformation provides the transfer function

$$
\frac{V_{q, d i f}(s)}{I_{v 1}(s)}=\frac{6 \cdot N \cdot v_{s t}}{V_{q} \cdot C_{s m} \cdot s}=\frac{6 \cdot v_{s t}}{V_{s m} \cdot C_{s m} \cdot s}
$$

In the case of the alternating current elements of the circulating currents, the model is developed using (14) but any other would result in the same. As in the previous case, the stored energy information of the branches related to the power $p_{34, c 1}$ is used

$$
p_{34, c 1}=\sqrt{3} \cdot v_{1, \beta} \cdot K_{u v} \cdot u_{v, 3}=\frac{\mathrm{d}}{\mathrm{d} t}\left(\frac{1}{2} \cdot \frac{C_{s m} \cdot v_{q_{34}}^{2}}{2 N}\right)
$$

where $v_{q_{34}}=v_{q 3}+v_{q 4}, K_{u v} \cdot u_{v, 3}=i_{v 3}$, and the system 1 peak value of any phase voltage is $V_{1, p k}$. In addition, it is assumed the operating point and small-signal perturbations $v_{q_{34}}=V_{q_{34}}+\tilde{v}_{q_{34}}$ and $i_{v 3}=I_{v 3}+\tilde{i}_{v 3}$. Thus,

$$
\sqrt{3} \cdot V_{1, p k} \cdot\left(I_{v 3}+\tilde{i}_{v 3}\right)=\frac{C_{s m}}{4 \cdot N} \cdot \frac{\mathrm{d}\left(2 \cdot V_{q 34} \cdot \tilde{v}_{q_{34}}+\tilde{v}_{q_{34}}^{2}\right)}{\mathrm{d} t}
$$

Once more the second order and dc elements are neglected. Applying the Laplace's transformation and extending the results for the other loops

$$
\begin{aligned}
& \frac{V_{q q}(s)}{I_{v 3}(s)}=\frac{V_{q q}(s)}{I_{v 2}(s)}=\frac{2 \cdot N \cdot \sqrt{3} \cdot V_{1, p k}}{V_{q q} \cdot C_{s m} \cdot s}=\frac{\sqrt{3} \cdot V_{1, p k}}{V_{s m} \cdot C_{s m} \cdot s} \\
& \frac{V_{q q}(s)}{I_{v 4}(s)}=\frac{V_{q q}(s)}{I_{v 5}(s)}=\frac{2 \cdot N \cdot \sqrt{3} \cdot V_{2, p k}}{V_{q q} \cdot C_{s m} \cdot s}=\frac{\sqrt{3} \cdot V_{2, p k}}{V_{s m} \cdot C_{s m} \cdot s}
\end{aligned}
$$

where $V_{q q}=V_{q_{34}}=V_{q 3}+V_{q 4}=2 \cdot N \cdot V_{s m}$.

\section{Discussion AND EXPERIMENTAL Results}

The presented three-phase ac-dc converter is tested according to the available hardware, which assembled set up is shown in Fig. 8. In total there are 24 full-bridge cells. The control platform is composed of a DSP and four FPGA boards. The DSP board has a Texas Instruments $225-\mathrm{MHz}$ TMS320C6713 which is responsible for executing the control laws and for the interface with a PC. While the FPGA (Actel ProAsic3 A3P400) boards are used for the data acquisition and PWM signal generation. Connected to the DSP board, it is equipped a daughter card for the interface and online data logging through a MATLAB host port interface (HPI).

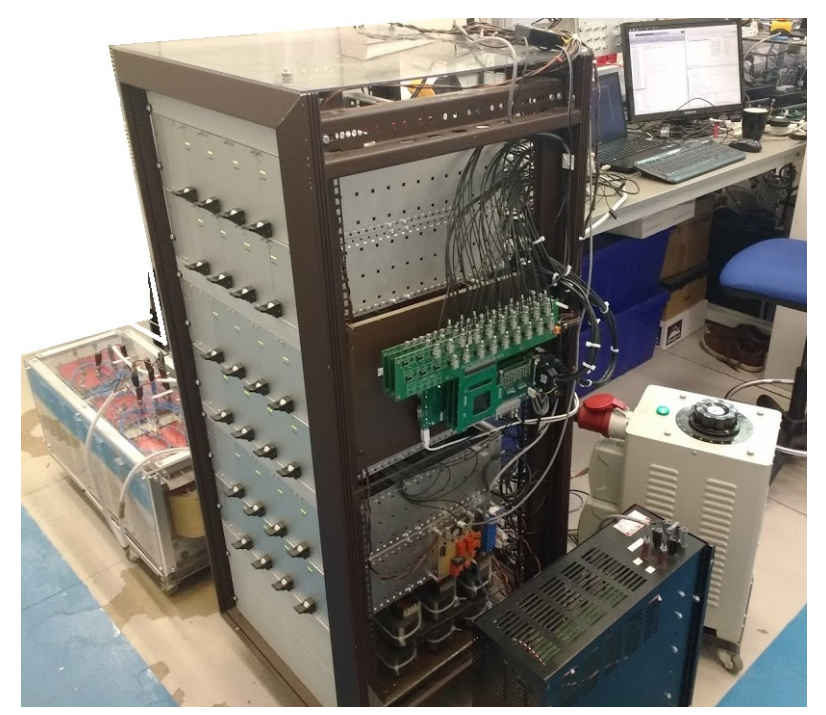

Fig. 8. Prototype in the rig (left to right 12-pulse rectifier, Hexverter, Variac and $\mathrm{PC}$ for the interface).

All the experiments parameters are shown in TABLE I where the given capacitance results in an $\mathrm{H}$ constant of approximately $60 \mathrm{~ms}$ [23]. Further than these, the DSP control loops and FPGA sampling frequencies are set to $7.2 \mathrm{kHz}$. The obtained results were captured with an oscilloscope Tektronix MSO 2024 but some internal variables were acquired FPGA.

TABLE I

CONVERTER PARAMETERS

\begin{tabular}{cl|cl}
\hline \hline Parameter & Value & Parameter & Value \\
\hline$f_{1}$ & $50 \mathrm{~Hz}$ & $f_{2}$ & $200 \mathrm{~Hz}$ \\
$L_{1}$ & $5 \mathrm{mH}$ & $L_{2}$ & $3 \mathrm{mH}$ \\
$L_{b}$ & $0.99 \mathrm{mH}$ & $C_{s m}$ & $300.8 \mu \mathrm{F}$ \\
$\mathrm{N}$ & 4 & $n_{12}$ & 2.79 \\
$v_{s m}$ & $150 \mathrm{~V}$ & $v_{s t}$ & $0.1 \cdot N \cdot v_{s m}$ \\
$v_{1, r m s}$ & $150 \mathrm{~V}$ & $v_{o}$ & $200 \mathrm{~V}$ \\
$R_{o}$ & $30.6 \Omega$ & $C_{o}$ & $1650 \mu \mathrm{F}$ \\
\hline \hline
\end{tabular}

The steady-state operation stability is verified through the controlled input currents, dc output voltage and SMs' voltages as shown in Fig. 9 and Fig. 10. These input current are ideally synchronized with the grid by the use of a phase-locked loop. In particular, it is shown by each branch modulation index in Fig. 11 that no saturation occurs under normal operation. 


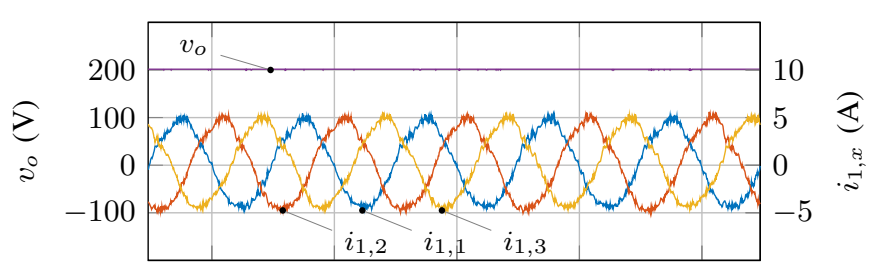

Time (20 ms/div)

Fig. 9. Input currents $\left(i_{1, x} x \in\{1,2,3\}\right)$ and output voltage $\left(v_{o}\right)$.

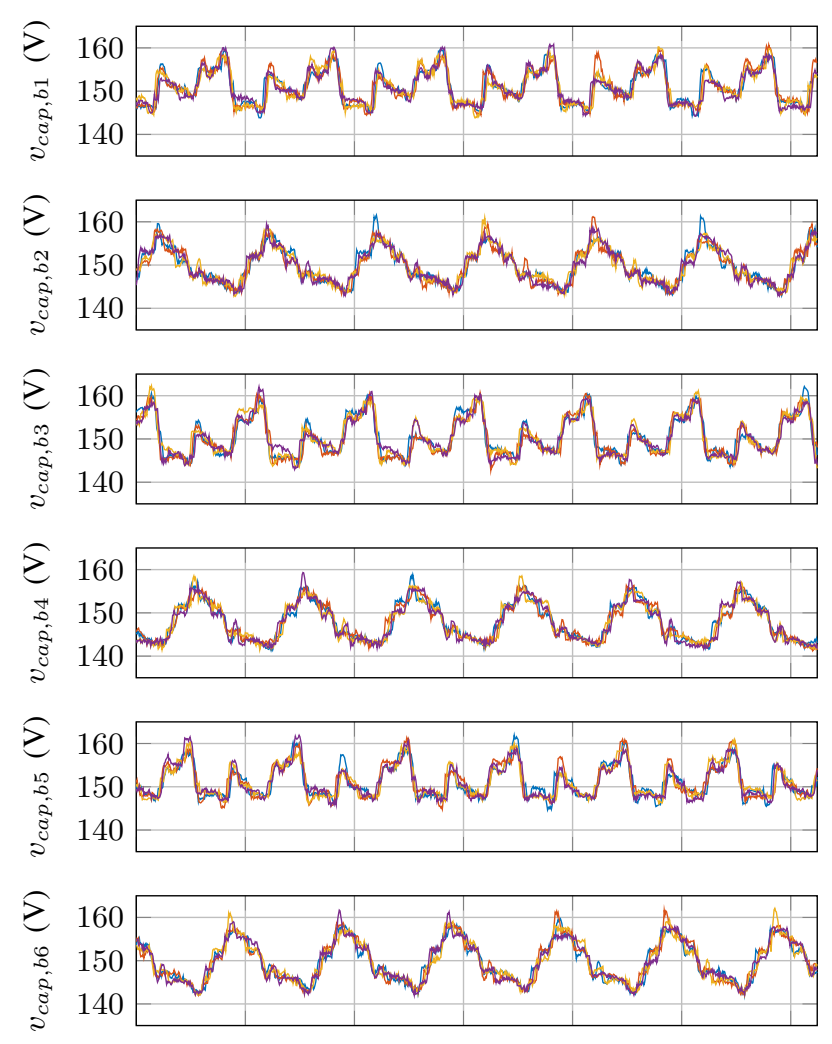

Time $(20 \mathrm{~ms} / \mathrm{div})$

Fig. 10. All the 24 submodule's capacitor voltages. In each graph, the four voltages of each branch are plotted.

The regulating branch energy loops control action $u_{v}$ are shown in Fig. 12. For the adjacent power loop (control action $\left.u_{v, 1}\right)$, the gain $K_{u v}$ is adjusted in order to provide a gain margin of $30.3 \mathrm{~dB}$ at $51.3 \mathrm{~Hz}$ and a phase-margin of $14.2^{\circ}$ at $8.81 \mathrm{~Hz}$. Note that the measured $v_{q, d i f}$ is filtered by a $2.5 \mathrm{~Hz}$ low-pass filter. The other regulating energy loops have a gain margin of $25 \mathrm{~dB}$ at $3.6 \mathrm{kHz}$ and a phase-margin of $88.4^{\circ}$ at $130 \mathrm{~Hz}$, which are the same because system 1 and system 2 phase voltages are ideally the same.

The proposed control strategy is verified by each loop main waveform. For the Hexverter current controllers, the $\alpha \beta$ variables are shown in Fig. 13 a) and Fig. 13 b), respectively. While the circulating current controller is shown in Fig. 13 c). Note that different than [14], our compensating power does not reach zero $\left(v_{s t} \cdot i_{c i r c}\right)$. The dc level in it indicates $Q_{1} \neq Q_{2}$ and the periodical oscillation indicates the nonactive power required by the 12-pulse rectifier due to the
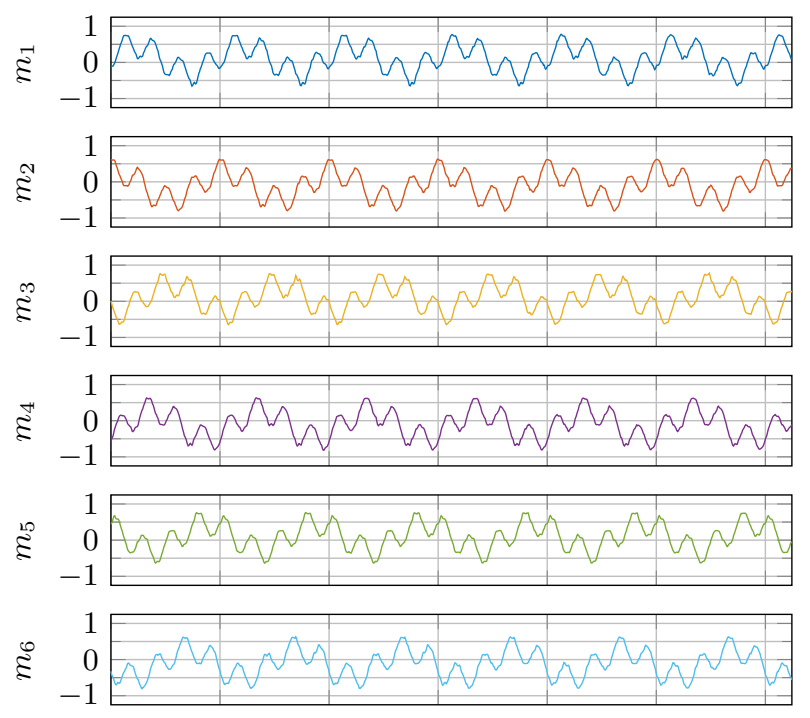

Time (20 ms/div)

Fig. 11. Each branch unitary index modulating signal $\left(m_{b} b \in\{1, \ldots 6\}\right)$.
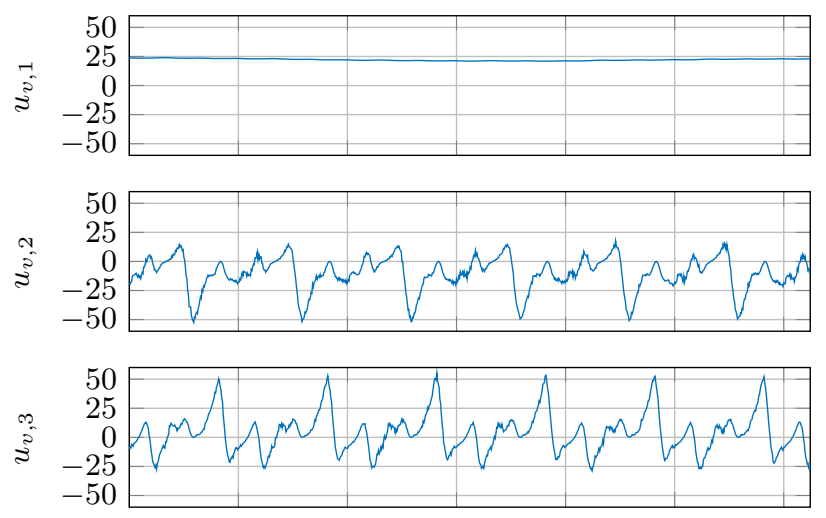

50
25
0
-25
-50 $\mathrm{~mm}$ W

25
20
-25
-50

Time (20 ms/div)

Fig. 12. Control actions of each loop in the Hexverter's branch energy control.

remaining harmonics. Both causes unbalance in the branch's energy.

System 1 and system 2 current controllers use a resonant plus proportional structure. While the circulating current controller is a proportional controller only. In all cases, the proportional controller is adjusted for the Bode Diagram zero cross frequency to be around $1.2 \mathrm{kHz}$, which is six times lower than the control law calculation frequency.

The converter stability is put under test by a fast stepup and an abrupt step-down of the output voltage reference. The intermediate voltage level is chosen in order to provide 


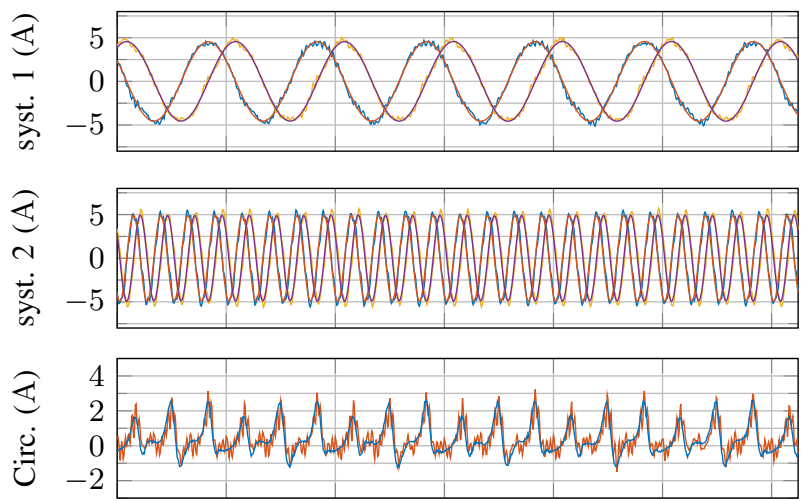

Time $(20 \mathrm{~ms} / \mathrm{div})$

Fig. 13. References and measured values (from top to bottom) for system 1 $\alpha \beta$ currents, system $2 \alpha \beta$ currents and the circulating current.

a power variation of $50 \%$. Both output voltage controller and the total voltage $\left(v_{t}\right)$ controller are proportional plus integral controllers. The sensed output voltage is filtered by a $20 \mathrm{~Hz}$ low-pass filter. While the total voltage is filtered by a $15 \mathrm{~Hz}$ low-pass filter. The output voltage controller is tuned to have a gain margin of $35.1 \mathrm{~dB}$ at $153 \mathrm{~Hz}$ and a phase-margin of $51.4^{\circ}$ at $16 \mathrm{~Hz}\left(\approx f_{2} / 10\right)$. While the total voltage controller is tuned to have a gain margin of $44.6 \mathrm{~dB}$ at $125 \mathrm{~Hz}$ and a phase-margin of $60.9^{\circ}$ at $6 \mathrm{~Hz}\left(\approx f_{1} / 10\right)$. The results of these controllers tunings are shown in Fig 14, where the overshoot and undershot occurred in accordance with the tunning.

a)
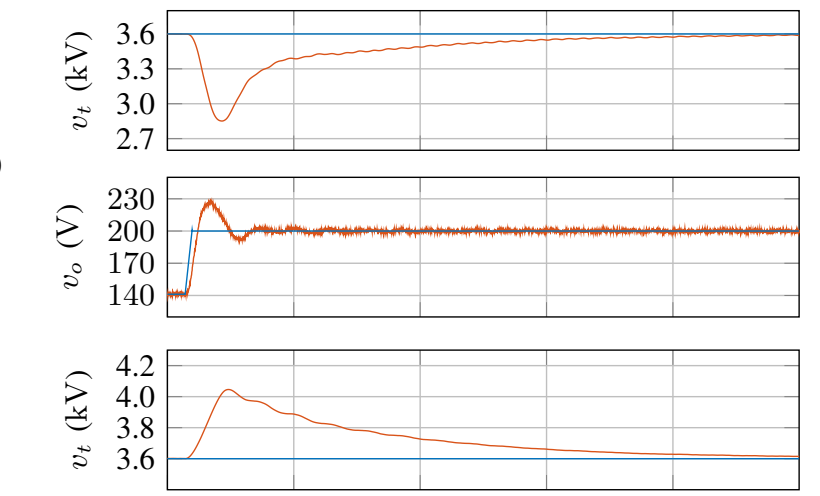

b)

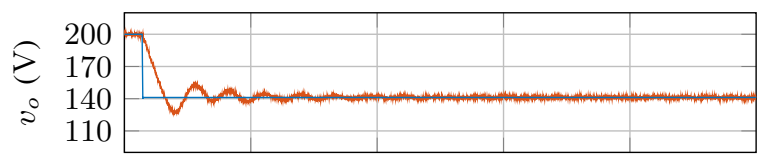

Time (100 ms/div)

Fig. 14. Transient response of the total voltage $\left(v_{t}\right)$ and the output voltage $\left(v_{o}\right)$ during step on the output voltage reference. Case a) step-up and case b) step-down.

Note that there is room for optimization in several variables. For instance, $i_{\text {circ }}$ could be reduced by setting higher $v_{s t}$ or by reducing the gain of the branch energy regulation loops. Also, each regulation loop could have different gains.

\section{CONCLUSION}

This paper has proposed a two-stage ac-dc converter which is the combination of a Hexverter and a series-type 12-pulse rectifier. A complete control strategy is presented in order to control the output voltage and the Hexverter stored energy. In particular, a new method for monitoring and action upon Hexverter's branch energy unbalance is explained and detailed. Experimental results verify the steady-state and transient stability of the converter and its control strategy.

Since the control strategy is based on the Hexverter's current control, the converter inherits short-circuit protection from the Hexverter point of view. Also, apart from the output filter and power line parasitic elements, even the rectifier current is limited under transient overloads. For instance, this feature eases the commissioning of its loads.

The energy regulation in all stages (even distribution and total value in Hexverter plus the output voltage) have been accomplished by the means of a simple control strategy. However, further analysis must be done when in the presence of a VSD (Variable Speed Drive) as a load. Also, transmission/distribution lines from the input or the output must be taken into consideration.

Finally, although one rectifier output is shown, the structure itself and the control strategy allows the insertion of multiple outputs by adding other windings in the transformer or another multipulse rectifier.

\section{REFERENCES}

[1] R. N. Fard and E. Tedeschi, "Integration of distributed energy resources into offshore and subsea grids," CPSS Transactions on Power Electronics and Applications, vol. 3, no. 1, pp. 36-45, March 2018.

[2] B. Westman, S. Gilje, and M. Hyttinen, "Valhall re-development project, power from shore," in PCIC Europe 2010, June 2010, pp. 1-5.

[3] K. Rajashekara and H. Krishnamoorthy, "Power electronics for subsea systems: Challenges and opportunities," in 2017 IEEE 12th International Conference on Power Electronics and Drive Systems (PEDS), Dec 2017, pp. $986-991$.

[4] T. Hazel, H. H. Baerd, J. J. Legeay, and J. J. Bremnes, "Taking power distribution under the sea: Design, manufacture, and assembly of a subsea electrical distribution system," IEEE Industry Applications Magazine, vol. 19, no. 5, pp. 58-67, Sept 2013.

[5] J. Song-Manguelle, M. H. Todorovic, S. Chi, S. K. Gunturi, and R. Datta, "Power transfer capability of hvac cables for subsea transmission and distribution systems," IEEE Trans. Ind. Appl., vol. 50, no. 4, pp. 2382 2391, July 2014.

[6] B. Monsen, K. S. Rongve, T. Lægreid, and C. Gutscher, "Åsgard subsea gas compression-technology qualification testing with high-speed vsd and very long step-out cable," IEEE Trans. Ind. Appl., vol. 50, no. 2, pp. 1552-1561, March 2014.

[7] H. Akagi, "A review of developments in the family of modular multilevel cascade converters," IEEJ Transactions on Electrical and Electronic Engineering, vol. 13, no. 9, pp. 1222-1235, 2018.

[8] S. Cui, J. Lee, J. Hu, R. W. De Doncker, and S. Sul, "A modular multilevel converter with a zigzag transformer for bipolar mvdc distribution systems," IEEE Trans. Power Electron., vol. 34, no. 2, pp. 1038-1043, Feb 2019.

[9] H. Nademi, A. Das, R. Burgos, and L. E. Norum, "A new circuit performance of modular multilevel inverter suitable for photovoltaic conversion plants," IEEE Journal of Emerging and Selected Topics in Power Electronics, vol. 4, no. 2, pp. 393-404, June 2016.

[10] K. Ilves, L. Bessegato, and S. Norrga, "Comparison of cascaded multilevel converter topologies for ac/ac conversion," in Power Electronics Conference (IPEC-Hiroshima 2014 - ECCE-ASIA), 2014 International, May 2014, pp. 1087-1094.

[11] D. Karwatzki, L. Baruschka, and A. Mertens, "Survey on the hexverter topology - a modular multilevel ac/ac converter," in Power Electronics and ECCE Asia (ICPE-ECCE Asia), 2015 9th International Conference on, June 2015, pp. 1075-1082.

[12] G. Lambert, M. V. Soares, P. Wheeler, M. L. Heldwein, and Y. R. de Novaes, "Current fed multipulse rectifier approach for unidirectional hvdc and mvdc applications," IEEE Trans. Power Electron., pp. 1-1, 2018. 
[13] F. Kammerer, J. Kolb, and M. Braun, "A novel cascaded vector control scheme for the modular multilevel matrix converter," in IECON 2011 37th Annual Conference of the IEEE Industrial Electronics Society, Nov 2011, pp. 1097-1102.

[14] D. Karwatzki, L. Baruschka, M. von Hofen, and A. Mertens, "Branch energy control for the modular multilevel direct converter hexverter," in Energy Conversion Congress and Exposition (ECCE), 2014 IEEE, Sept 2014, pp. 1613-1622.

[15] L. Baruschka and A. Mertens, "A new 3-phase direct modular multilevel converter," in Power Electronics and Applications (EPE 2011), Proceedings of the 2011-14th European Conference on, Aug 2011, pp. 1-10.

[16] _ "A new three-phase ac/ac modular multilevel converter with six branches in hexagonal configuration," IEEE Trans. Ind. Appl., vol. 49, no. 3, pp. 1400-1410, May 2013.

[17] H. Akagi, "Classification, terminology, and application of the modular multilevel cascade converter (mmcc)," IEEE Trans. Power Electron., vol. 26, no. 11, pp. 3119-3130, Nov 2011.

[18] S. Yang, Y. Tang, and P. Wang, "Distributed control for a modular multilevel converter," IEEE Trans. Power Electron., vol. 33, no. 7, pp. 5578-5591, July 2018.

[19] A. Dekka, B. Wu, N. R. Zargari, and R. L. Fuentes, "Dynamic voltage balancing algorithm for modular multilevel converter: A unique solution," IEEE Trans. Power Electron., vol. 31, no. 2, pp. 952-963, Feb 2016.

[20] H. Peng, R. Xie, K. Wang, Y. Deng, X. He, and R. Zhao, "A capacitor voltage balancing method with fundamental sorting frequency for modular multilevel converters under staircase modulation," IEEE Trans. Power Electron., vol. 31, no. 11, pp. 7809-7822, Nov 2016.

[21] D. Karwatzki, L. Baruschka, M. von Hofen, and A. Mertens, "Optimised operation mode for the hexverter topology based on adjacent compensating power," in 2014 IEEE Energy Conversion Congress and Exposition (ECCE), Sept 2014, pp. 5399-5406.

[22] G. Lambert, M. L. Heldwein, and Y. R. de Novaes, "Simplified modeling and control of a high-power high-voltage isolated dc-dc converter," in 2015 IEEE 13th Brazilian Power Electronics Conference and 1st Southern Power Electronics Conference (COBEP/SPEC), Nov 2015, pp. $1-6$.

[23] A. Suzuki and H. Akagi, "Hvdc circuit breakers combining mechanical switches and a multilevel pwm converter: Verification by downscaled models," IEEE Trans. Power Electron., vol. 34, no. 5, pp. 4259-4269, May 2019.

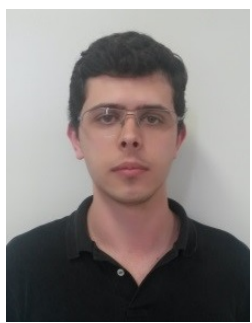

Gustavo Lambert (S"14) received the B.S. and M.S. degrees in electrical engineering from the Santa Catarina State University (UDESC), Joinville, Brazil, in 2012 and 2015, respectively. He received the Dr. degree from the Santa Catarina State University in 2019 for his work on Modular Multilevel Converters. Currently, he is a postdoctoral researcher at nPEE (Electric Power Processing Group), Santa Catarina State University. His research interests include the design and control of modular multilevel converter topologies for HVDC and MVDC applications.

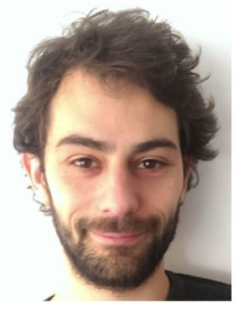

Alessandro Costabeber (S'09-M'13) received the Degree with honours in Electronic Engineering from the University of Padova, Padova, Italy, in 2008 and the Ph.D. in Information Engineering from the same university in 2012, on energy efficient architectures and control techniques for the development of future residential microgrids. In the same year he started a two-year research fellowship with the same university. In 2014 he joined the PEMC group, Department of Electrical and Electronic Engineering, University of Nottingham, Nottingham, UK as lecturer in Power Electronics. His current research interests include HVDC converters topologies, high power density converters for aerospace applications, control solutions and stability analysis of AC and DC microgrids, control and modeling of power converters, power electronics and control for distributed and renewable energy sources. Dr. Costabeber received the IEEE Joseph John Suozzi INTELEC Fellowship Award in Power Electronics in 2011.

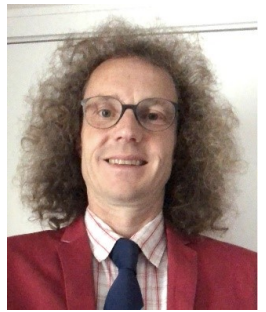

Patrick Wheeler (M'00-SM'12) received his BEng [Hons] degree in 1990 from the University of Bristol, UK. He received his $\mathrm{PhD}$ degree in Electrical Engineering for his work on Matrix Converters from the University of Bristol, UK in 1994. In 1993 he moved to the University of Nottingham and worked as a research assistant in the Department of Electrical and Electronic Engineering. In 1996 he became a Lecturer in the Power Electronics, Machines and Control Group at the University of Nottingham, UK. Since January 2008 he has been a Full Professor in the same research group. He was Head of the Department of Electrical and Electronic Engineering at the University of Nottingham from 2015 to 2018. $\mathrm{He}$ is currently the Head of the Power Electronics, Machines and Control Research Group, Global Director of the University of Nottingham's Institute of Aerospace Technology and is the Li Dak Sum Chair Professor in Electrical and Aerospace Engineering. He is a member of the IEEE PELs AdCom and was an IEEE PELs Distinguished Lecturer from 2013 to 2017. He has published over 600 academic publications in leading international conferences and journals.

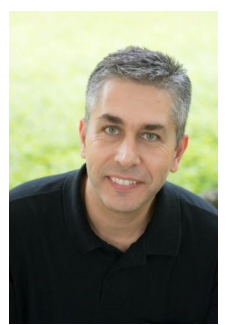

Yales Romulo De Novaes Yales Rômulo De Novaes (IEEE member) was born in Indaial, Santa Catarina, Brazil, in 1974. In 1999 he received the B.S. degree in electrical engineering from FURB - Regional University of Blumenau, Santa Catarina, Brazil. The M. Eng. and Dr. degrees were obtained from the Power Electronics Institute (INEP) of the Federal University of Santa Catarina (UFSC) in 2000 and 2006, respectively. During 2001, he worked as a researcher engineer at the same institute. From 2006 to 2008 was a post-doctoral fellow at the Industrial Electronics Laboratory (LEI) at École Polytechnique Fédéral de Lausanne (EPFL), Lausanne, Switzerland. From 2008 to 2010 worked as scientist with the Power Electronics Systems group at ABB Corporate Research Center, Daetwill, Switzerland. Currently he is an associate professor at Santa Catarina State University (UDESC), Joinville, Brazil, and coordinator of CMEAR (Research Cell on Microgrids of Alternative and Renewable Energies). 